Sitzungsberichte

der Heidelberger Akademie der Wissenschaften

Mathematisch-naturwissenschaftliche Klasse

Jahrgang 1960/1961, 4. Abhandlung

\title{
Projektive Frobenius-Erweiterungen
}

$$
\text { Von }
$$

Friedrich Kasch

(Vorgelegt in der Sitzung vom 10. November 1960)
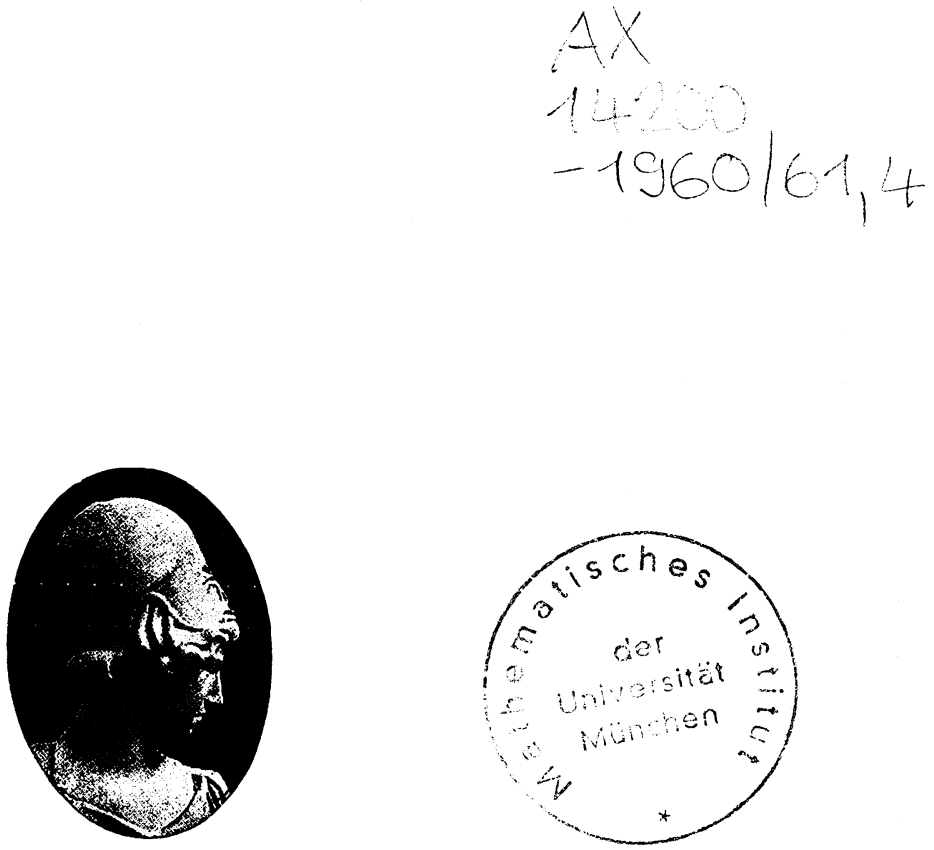

Heidelberg 1961

S p ringer-Verlag 


\title{
Projektive Frobenius=Erweiterungen
}

Von

\author{
Friedrich Kasch, Heidelberg
}

Vorgelegt in der Sitzung vom 10. November 1960

\section{Einleitung}

Eine Frobenius-Algebra ist bekanntlich eine endlichdimensionale Algebra $\Gamma / \Lambda$ mit 1-Element, für die ein $\Gamma$-Modulisomorphismus

$$
\Gamma \simeq \operatorname{Hom}_{\Lambda}(\Gamma, \Lambda)
$$

existiert. Diese Dualitätseigenschaft hat man von den Gruppenringen endlicher Gruppen übernommen.

Frobenius-Algebren sind in zwei verschiedenen Richtungen verallgemeinert worden. Einerseits zu Frobenius- und Quasi-Frobenius-Ringen, bei denen man die Bezugnahme auf einen Unterkörper oder Unterring $\Lambda$ fallen gelassen hat. Andererseits hat man diese Bezugnahme und die Voraussetzung (1) beibehalten, aber die sonstigen Voraussetzungen abgeschwächt.

So betrachten EilenberG-Nakayama in [3] Frobenius-Algebren $\Gamma$ über einem Ring $A$, wobei die Voraussetzung der endlichen Dimension im Falle eines Körpers $\Lambda$ durch die Forderung, da $\Gamma$ als $\Lambda$-Modul endlich erzeugt und projektiv sei, ersetzt wird. In [3] werden homologische Eigenschaften derartiger Algebren untersucht.

Ferner habe ich in [9] den Begriff der Frobenius-Erweiterung eingeführt, der kürzlich von NAKAYAMA-TSUzUkU in [11] von gewissen Einschränkungen befreit wurde und sich nach [11] folgendermaßen darstellt: Es seien $I$ ein Ring mit 1-Element, $A$ ein Unterring mit dem gleichen 1 -Element, es sei $\Gamma$ als $\Lambda$-Rechtsmodul endlich erzeugt und frei, und es gelte (1) als $A-I$-Isomorphie.

Auf der Grundlage von [9] hat K. Hirata [7] homologische Eigenschaften von Frobenius-Erweiterungen untersucht. Es ist nun wünschenswert, diese Resultate und die von EILENBERG-NAKAYAMA [3] möglichst einheitlich zu gewinuen. Dazu schwächen wir den Begriff der Frobenius-Erweiterung dahingehend $a b$, da $B$ wir bei 
sonst gleichen Voraussetzungen an Stelle von „frei“ nur ,,projektiv" verlangen. Damit haben wir die im Titel genannten projektiven Frobenius-Erweiterungen erhalten.

Im ersten Teil der Arbeit zeigen wir, daß die Definition der projektiven Frobenius-Erweiterungen symmetrisch ist und stellen Hilfsmittel für die weiteren Überlegungen bereit. Insbesondere zeigen wir, daß es projektive Frobenius-Erweiterungen gibt, die nicht frei sind.

Im zweiten Teil wird untersucht, wie weit sich eine FrobeniusErweiterung durch ihren $A$-Endomorphismenring $\operatorname{Hom}_{.1}(T, T)$ charakterisieren läßt. Hier werden wir einen Satz, der nach [9, $11,10]$ für freie Frobenius-Erweiterungen bekannt ist, auf projektive Frobenius-Erweiterungen verallgemeinern.

Im dritten Teil betrachten wir dann die homologischen Eigenschaften von projektiven Frobenius-Erweiterungen und erhalten zunächst Resultate, die Ergebnisse von EILENBERG-NAKAyama [3] und K. Hirata [7] enthalten und ergänzen.

Sodann schränken wir die Frobenius-Erweiterungen auf ausgezeichnete projektive Frobenius-Erweiterungen ein. Dabei heiße $I / \Lambda$ ausgezeichnet, wenn $\Gamma$ als zweiseitiger $A$-Modul einen zu $A$ isomorphen direkten Summanden besitzt. Hier ergibt sich als Hauptresultat, daß die schwache bzw. projektive bzw. injektive Dimension eines $A$-Moduls $A$ mit der entsprechenden Dimension des $\Gamma$-Moduls $A \otimes \Gamma$ und $\operatorname{Hom}_{A}(\Gamma, A)$ übereinstimmt.

Schlieljlich zeigen wir für freie Frobenius-Erweiterungen, wo eine Spurbildüng möglich ist, daß die durch die Spur eines $A$-Homomorphismus in den $\operatorname{Ext}_{(I, A)}^{i}$ für $i>0$ induzierte Abbildung jeweils die Nullabbildung ist. Dieses Resultat, das für Gruppenringe bekannt ist, besitzt interessante Folgerungen.

\section{Definition und Kennzeichnung von Frobenius-Erweiterungen}

\subsection{Voraussetzungen}

Es seien in dieser Arbeit stets $\Gamma$ ein Ring mit 1-Element und .1 ein Unterring von $\Gamma$ mit dem gleichen 1-Element. Alle $I^{\prime}$ - und $A$-Moduln seien unitär. Ist $A$ ein $\Gamma$-Rechts- bzw. $I$-Linksmodul, so schreiben wir auch $A_{\Gamma}$ bzw. ${ }_{T} A$. Die Schreibweise ${ }_{1} A_{1} \sim{ }_{1} E_{1}$ bedeute, da $\beta$ die $I$-Links- und $A$-Rechtsmoduln $A$ und $B I$ - $A$-isomorph seien. Bei Rechtsmoduln schreiben wir Abbildungen links von dem abzubildenden Element und bei Linksmoduln rechts; ist 
z. B. $f$ eine Abbildung von $A_{\Gamma}$ bzw. ${ }_{T} A$, dann sei $f(a)$ bzw. $(a) f$ das Bild eines Elementes $a \in A$ bei $f$. Der Modul $\operatorname{Hom}_{1}\left(A_{A}, \Gamma_{A}\right)$ wird zu einem $\Gamma$-Linksmodul, wenn $(\gamma f)(a)=\gamma \dot{f}(a)$ für $f \in \operatorname{Hom}_{A}\left(A_{.1}, \Gamma_{.1}\right)$, $\gamma \in \Gamma, a \in A$ gesetzt wird. Entsprechend wird $\operatorname{Hom}_{.}\left(\Gamma_{1}, A_{.1}\right)$ zu einem $\Gamma$-Rechtsmodul durch die Festsetzung $(f \gamma)(\xi)=f(\gamma \xi)$ für $f \in \operatorname{Hom}_{.1}\left(\Gamma_{.1}, A_{.1}\right)$ und $\gamma, \xi \in \Gamma$.

Sei jetzt

$$
F_{A}=\bigoplus_{i=1}^{n} x_{i} A
$$

ein freier $A$-Rechtsmodul mit den freien Erzeugenden (= Basis) $x_{1}$, $\ldots, x_{n}$. Ist jeweils für $i=1, \ldots, n$ die Abbildung $d_{i} \in \operatorname{Hom}_{.1}\left(F_{.1}, \Gamma_{A}\right)$ durch

$$
d_{i}\left(x_{j}\right)=\delta_{i j}= \begin{cases}0 & \text { für } i \neq j \\ 1 & \text { für } i=j\end{cases}
$$

definiert, dann gilt offenbar

und ebenso

$$
\operatorname{Hom}_{A}\left(F_{A}, \Gamma_{A}\right)=\bigoplus_{i=1}^{n} \Gamma d_{i}
$$

Ferner folgt

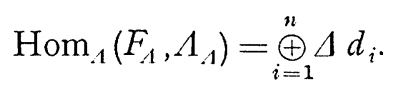

$$
\Gamma \operatorname{Hom}_{A}\left(F_{A}, A_{A}\right)=\operatorname{Hom}_{A}\left(F_{A}, \Gamma_{.1}\right) .
$$

Sei nun $F_{A}=A_{.1} \oplus B_{1}$, dann denken wir uns jede Abbildung $f=\operatorname{Hom}_{A}\left(A_{A}, \Gamma_{.1}\right)$ durch die Festsetzung $f(b)=0$ für alle $b \in B$ zu einer Abbildung von $F_{A}$ fortgesetzt, so daß $\operatorname{Hom}_{.1}\left(A_{1}, \Gamma_{.1}\right)$ als Untermodul von $\operatorname{Hom}_{A}\left(F_{A}, \Gamma_{A}\right)$ betrachtet werden kann; entsprechend für $B_{1}$. Dann gelten die folgenden Gleichungen:

$$
\left.\begin{array}{rl}
\operatorname{Hom}_{A}\left(F_{.1}, \Gamma_{A}\right) & =\operatorname{Hom}_{A}\left(A_{.1}, \Gamma_{.}\right) \oplus \operatorname{Hom}_{.1}\left(B_{.1}, \Gamma_{.}\right) \\
\operatorname{Hom}_{.1}\left(F_{.1}, A_{.1}\right) & =\operatorname{Hom}_{.1}\left(A_{.1}, A_{.1}\right) \oplus \operatorname{Hom}_{.1}\left(B_{.1}, A_{.1}\right) \\
\operatorname{Hom}_{.1}\left(A_{.1}, A_{A}\right) & =\operatorname{Hom}_{A}\left(A_{A}, \Gamma_{.1}\right) .
\end{array}\right\}
$$

Ferner besitzt $\operatorname{Hom}_{A}\left(A_{A}, \Gamma_{A}\right)$ bzw. $\operatorname{Hom}_{1}\left(A_{1}, A_{1}\right)$ als $\Gamma$ - bzw. $A$-Linksmodul ein endliches Erzeugendensystem, nämlich die Einschränkungen der Abbildungen $d_{i}(i=1, \ldots, n)$ von $F$ auf $A$.

Sei jetzt $\Gamma$ als $A$-Rechtsmodul endlich erzeugt und projektiv, dann ist $\Gamma$ direkter Summand eines endlich erzeugten freien $A$-Rechtsmoduls $F_{A}$, und für $\Gamma_{A}$ treffen die zuvor für $A_{.1}$ gemachten Feststellungen zu. Daraus folgt, da $B \operatorname{Hom}_{.1}\left(\Gamma_{1}, \Lambda_{.1}\right)$ als $\Lambda$-Linksmodul ebenfalls endlich erzeugt und projektiv ist. Außerdem ist zu be- 
merken, daß $\operatorname{Hom}_{.1}\left(\Gamma_{.1}, A_{.1}\right)$ nicht nur $A$-Links- sondern auch noch $I$-Rechtsmodul ist.

Da $\Gamma_{.1}$ endlich erzeugt und projektiv ist, gilt für jeden Modul ${ }_{1} C$ (nach [3], S. 2)

$$
\Gamma \otimes \underset{A}{\otimes} C \simeq \operatorname{Hom}_{A}\left({ }_{A} \operatorname{Hom}_{.1}\left(\Gamma_{A}, A_{A}\right),{ }_{.1} C\right)
$$

als $\Gamma$-Linksmoduln; ebenso gilt für jeden Modul $C_{A}$

$$
C \otimes \Gamma \simeq \operatorname{Hom}_{A}\left(\operatorname{Hom}_{A}\left({ }_{A} \Gamma,{ }_{1} \Lambda\right)_{A}, C_{A}\right)
$$

als $\Gamma$-Rechtsmoduln. Aus (5) folgt speziell für $C=\Lambda$ die $I$ - $A$-Isomorphie

$$
\Gamma \cong \operatorname{Hom}_{\Lambda}\left({ }_{\Lambda} \operatorname{Hom}_{A}\left(\Gamma_{A}, \Lambda_{A}\right),{ }_{\Lambda} \Lambda\right),
$$

die explizit durch

$$
\Gamma \ni \gamma \rightarrow\left(\operatorname{Hom}_{\Lambda}\left(\Gamma_{\Lambda}, \Lambda_{A}\right) \ni t \rightarrow f(\gamma) \in \Lambda\right)
$$

gegeben wird.

\subsection{Definition der Frobenius-Erweiterungen}

Wir gehen von den folgenden Bedingungen aus:

(r1) ${ }_{A} \Gamma_{\Gamma} \cong{ }_{A} \operatorname{Hom}_{A}\left(\Gamma_{A}, \Lambda_{A}\right)_{\Gamma}$

(r2) $\quad \Gamma_{A}$ ist endlich erzeugt und projektiv

(r3) $\quad \Gamma_{A}$ ist endlich erzeugt und frei

(l1) $\quad{ }_{T} \Gamma_{A} \simeq{ }_{\Gamma} \operatorname{Hom}_{A}\left({ }_{A} \Gamma,{ }_{A} \Lambda\right)_{A}$

(12) ${ }_{A} \Gamma$ ist endlich erzeugt und projektiv

(13) ${ }_{4} \Gamma$ ist endlich erzeugt und frei.

\section{Bemerkung 1}

a) Die Bedingungen ( $\mathrm{r} 1$ ) und ( $\mathrm{r} 2$ ) sind äquivalent zu (11) und (12).

b) Die Bedingungen ( $\mathrm{r} 1$ ) und ( $\mathrm{r} 3$ ) sind äquivalent $z u$ (11) und (13).

Beweis. Seien (r1) und (r2) erfüllt. Wie schon festgestellt, ist wegen (r 2) $\operatorname{Hom}_{A}\left(\Gamma_{A}, \Lambda_{A}\right)$ als $\Lambda$-Linksmodul endlich erzeugt und projektiv. Wegen (r1) folgt dann (12). Gilt (r3), so erhält man ebenso (13). Wegen ( $\mathrm{r} 1$ ) und (7) gilt schließlich (11). Die Umkehrung folgt ebenso.

\section{Definition}

a) Die Ringerweiterung $\Gamma / \Lambda$ heißt Frobenius-Erweiterung, wenn die Bedingungen aus Bemerkung 1a) erfüllt sind. 
b) Die Ringerweiterung $I / A$ heißt freie Frobenius-Erweiterung, wenn die Bedingungen aus Bemerkung 1b) erfiillt sind.

Die im Titel dieser Arbeit genannten projektiven FrobeniusErweiterungen werden also jetzt kurz Frobenius-Erweiterungen genannt, und nur die spezielleren freien Frobenius-Erweiterungen crhalten das zusätzliche Adjektiv.

Wir bemerken schließlich noch, daß es zu je zwei Isomorphismen $\varphi_{1}$ und $\varphi_{2}$ von ${ }_{A} \Gamma_{\Gamma}$ und ${ }_{A} \operatorname{Hom}_{A}\left(\Gamma_{1}, A_{1}\right)_{\Gamma}$ stets ein invertierbares Element $\zeta$ aus dem Zentralisator von $A$ in $I$ gibt, so da $\beta$ $\varphi_{2}(\gamma)=\varphi_{1}(\zeta \gamma)$ für alle $\gamma \in I$ gilt.

Wie bei freien Frobenius-Erweiterungen kann auch jetzt der Isomorphismus ( $\mathrm{r} 1$ ) bzw. (11), den wir auch als Frobenius-Isomorphismus bezeichnen wollen, durch einen ,Frobenius-Homomorphismus" ersetzt werden. Dazu betrachten wir die folgenden Bedingungen:

$\left(\mathrm{r} 1^{\prime}\right)$ Es gibt einen zweiseitigen $A$-Homomorphismus $\psi$ von $\Gamma$ in 1 so, daß die Abbildung

$$
\Gamma \ni \gamma \rightarrow \psi \gamma=\operatorname{Hom}_{.1}\left(\Gamma_{.1}, \Lambda_{.1}\right)
$$

ein $A$ - $T$-Isomorphismus ist.

$\left(11^{\prime}\right)$ Es gibt einen zweiseitigen 1 -Homomorphismus $\psi$ von $\Gamma$ in 1 so, daß die Abbildung

$$
\Gamma \doteq \gamma \rightarrow \gamma \psi \in \operatorname{Hom}_{A}\left(.{ }_{.1} \Gamma,{ }_{.1} 1\right)
$$

ein $\Gamma$ - $\Lambda$-Isomorphismus ist.

Bemerkung 2. Dann und nur dann ist $\Gamma / 1$ eine Frobeniuserweiterung bzw. eine treie Frobenius-Erweiterung, wenn ( $\left.\mathrm{r} 1^{\prime}\right)$ und

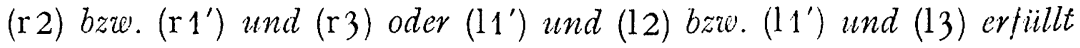
sind.

Beweis. Es genügt zu zeigen, daß ( $\mathrm{r} 1)$ und $\left(\mathrm{r} 1^{\prime}\right)$ äquivalent sind. Aus $\left(\mathrm{r}^{\prime}{ }^{\prime}\right)$ folgt unmittelbar ( $\left.\mathrm{r} 1\right)$. Sei nun ( $\left.\mathrm{r} 1\right)$ erfiullt und $\varphi$ der 1- $\Gamma$-Isomorphismus von $\Gamma$ und $\operatorname{Hom}_{.1}\left(\Gamma_{1}, \Lambda_{1}\right)$, dann wollen wir zeigen, daß $\psi=\varphi(1)$ die Bedingung $\left(\mathrm{r}^{\prime}\right)$ erfüllt. Als Element aus $\operatorname{Hom}_{1}\left(\Gamma_{A}, \Lambda_{1}\right)$ ist $\psi$ ein $A$-Rechtshomomorphismus. Ferner gilt für $\lambda \in A, \gamma \in \Gamma$

$$
\psi(\lambda \gamma)=\varphi(1)(\lambda \gamma)=\varphi(\lambda)(\gamma)=\lambda \varphi(1)(\gamma)=\lambda \psi \psi(\gamma),
$$

also ist $\psi$ ein zweiseitiger 1 -Homomorphismus. Die Abbildung

$$
\Gamma \ni \gamma \rightarrow \psi \gamma=\varphi(1) \gamma=\varphi(\gamma) \in \operatorname{Hom}_{.1}\left(\Gamma_{1}, A_{A}\right)
$$


stimmt mit $\varphi$ überein und ist folglich ein $\Lambda-I$ '-Isomorphisnnuuulus. Damit ist $\left(\mathrm{r} 1^{\prime}\right)$ bewiesen.

Wir stellen noch fest, daß bei einer freen Frobenius-Erweiititcerung $\psi$ ein Epimorphismus ist. $Z u$ jedem $\lambda \in \Lambda$ gibt es dann näinimlich (mindestens) ein $h \in \operatorname{Hom}_{A}\left(\Gamma_{A}, \Lambda_{A}\right)$ und ein $\gamma \in \Gamma$ mit $h(\gamma)==, ;, \lambda$; sei $\psi \gamma_{0}=h$, dann folgt $\psi\left(\gamma_{0} \gamma\right)=\lambda$.

Schließlich wollen wir feststellen, daß es Frobenius-Erweiterumungen gibt, die nicht frei sind. Dazu betrachten wir zu zwei Frotbiboenius-Erweiterungen $\Gamma_{1} / \Lambda_{1}$ und $\Gamma_{2} / \Lambda_{2}$ die direkte Summe $I=\Gamma_{1} \Theta 11 \Gamma_{2}$, für die die Multiplikation durch $\left(\gamma_{1}+\gamma_{2}\right)\left(\gamma_{1}^{\prime}+\gamma_{2}^{\prime}\right)=\gamma_{1} \gamma_{1}^{\prime}+\gamma_{2}: \gamma_{1}^{\prime} \gamma_{2}^{\prime}$, $\gamma_{1}, \gamma_{1}^{\prime} \in \Gamma_{1}, \gamma_{2}, \gamma_{2}^{\prime} \in \Gamma_{2}$ definiert sei. Dann ist $\Gamma$ ein Ring urumd $\Lambda=\Lambda_{1} \oplus \Lambda_{2}$ ein Unterring von $\Gamma$ mit dem gleichen 1-Elemeen'mit. Man prüft sofort nach, da $\Omega / \Lambda$ Frobenius-Erweiterung ist. Auß3ejezrdem ergibt sich, daß $\Gamma / \Lambda$ dann und nur dann freie Frobenius-]EEirweiterung ist, wenn $T_{1} / A_{1}$ und $\Gamma_{2} / A_{2}$ freie Frobenius-Erweiterungegecen gleicher Dimension sind. Daraus folgt sofort, daß es FrobeniiuunsErweiterungen gibt, die nicht frei sind.

\subsection{Freie Frobenius-Erweiterungen}

Wir stellen hier einige (aus [9] und [11]) bekannte Tatsachecen über freie Frobenius-Erweiterungen zusammen, die später ggsebraucht werden.

Sei $\Gamma / \Lambda$ eine Ringerweiterung und seien $r_{1}, \ldots, r_{n}$ eine Rechitittssowie $l_{1}, \ldots, l_{n}$ eine Linksbasis von $\Gamma / \Lambda$. Diese Basen heißen d:u uxal (zueinander), wenn die durch $r_{1}, \ldots, r_{n}$ erzeugte Rechtsdarstelliurımg von $\Gamma$ in $A_{n}$ mit der durch $l_{1}, \ldots, l_{n}$ erzeugten Linksdarstellurumg übereinstimmt. Das bedeutet, da $\beta$ für jedes $\gamma \in \Gamma$ aus

$$
\gamma r_{j}=\sum_{i=1}^{n} r_{i} \lambda_{i j}, \quad \lambda_{i j} \in \Lambda \quad(j=1, \ldots, n)
$$

die Gleichungen

$$
l_{i} \gamma=\sum_{i=1}^{n} \lambda_{i i} l_{j} \quad(i=1, \ldots, n)
$$

folgen und umgekehrt.

Wesentlich ist nun, da $\beta$ freie Frobenius-Erweiterungen durrrcch duale Basen charakterisiert werden können. Es gilt: Dann itvınd nur dann ist $\Gamma / \Lambda$ eine freie Frobenius-Erweiterung, wenn endlicicihi duale Basen von $\Gamma / \Delta$ existieren.

Mit Hilfe von dualen Basen kann nun auch sofort ein FrobeniuuusHomomorphismus $\psi$ angegeben werden, der gleichzeitig ( $\left.\mathrm{r} 1^{\prime}\right)$ urmmd 


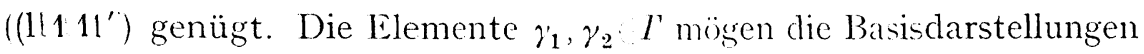

$$
\gamma_{1}=\sum_{i=1}^{n} \lambda_{i} l_{i}, \quad \gamma_{2}=\sum_{i=1}^{n} r_{i},_{i}, \quad \lambda_{i}, \mu_{i} A
$$

Ibvesessitzen, dann wird $\psi$ für das Produkt $\gamma_{1} \gamma_{2}$ durch

$$
\psi\left(\gamma_{1} \gamma_{2}\right)=\sum_{i=1}^{n} \lambda_{i} \prime_{i}
$$

, dleiesfiniert, so daß insbesondere

$$
\psi\left(l_{i} r_{j}\right)=\delta_{i j}
$$

grilillt. Da man jedes Element $\gamma /$ als Produkt von zwei Elementen siciclhreiben kann (z.B. $\gamma=1 \gamma$ ) und da $\psi\left(\gamma_{1} \gamma_{2}\right)$ wegen der Dualität dlele?r Basen nur vom Produkt abhängt, licfert $\psi$ einen Homomorphismnius von $\Gamma$ in $A$, der gleichzeitig $\left(\mathrm{r}^{\prime}\right)$ und $\left(11^{\prime}\right)$ erfüllt.

Für später merken wir noch die Gleichung

$$
1=\sum_{i=1}^{n} \psi\left(r_{i}\right) l_{i}
$$

annm, die sofort aus (8) folgt.

\section{Kennzeichnung einer Frobenius-Erweiternng durch ihren Endomorphismenring}

2.1. In [9] habe ich den besonders im Hinblick auf die Galoiss;cic:he Theorie der Schiefkörper und Ringe interessierenden folgenden ¿Ssatz bewiesen: Unter gewissen Voraussetzungen (die hier nicht annigegeben werden sollen) ist eine endlich erzeugte, freie Ring(errweiterung $I / A$ dann und nur dann (frcie) Frobenius-Erweiterung, wwwem der 1 -Endomorphismenring $\operatorname{Hom}_{i 1}\left(I_{1}, I_{.1}\right)$ Frobenius-Er'wweiterung des Ringes $I^{l}$ der Linksmultiplikatoren ${ }^{\prime}$ ' $l$ ist. KürzJlidich konnten NaKayama-Ts[zukU in [11] reigen, daß die dabei won mir gemachten Voraussetzungen überflüssig sind. Schließlich lkkonnte ich in [10] einen Beweis dieses Satzes für freie FrobeniusEE rweiterungen geben, bei dem nicht von dualen Basen Gebrauch : grgemacht wird. Diese Beweisführung ermöglicht is nun, den Satz nmit einer gewissen Einschränkung auch für beliclige FrobeniusEErweiterungen zu beweisen.

2.2. Zur Vorbereitung beweisen wir drei Hilfssätze.

Hilfssatz 1: Sei $\Gamma / \Lambda$ eine beliebige Ringerweiternng. Für jedes $1 t=\operatorname{Hom}_{.1}\left(\Gamma_{.1}, \Lambda_{.1}\right)$ gilt dann $\mathrm{Hom}_{.1}\left(\Gamma_{.1}, \Gamma_{.1}\right) t=\Gamma t$. 
Beweis. Für jedes $\xi \in T$ gilt $f(\xi) \in \Lambda$; daher folgt für beliebiges $h \in \operatorname{Hom}_{.1}\left(\Gamma_{A}, \Gamma_{A}\right)$

$$
h f(\xi)=h(1+(\xi))=h(1) f(\xi)=(h(1) f)(\xi) .
$$

Hilfssatz 2: Sei $\Gamma / \Lambda$ eine Ringerweiterung, und sei $\Gamma_{.1}$ projektiv. Dann gibt es zu jedem $h \in \operatorname{Hom}_{1}\left(\Gamma_{A}, \Gamma_{A}\right), h \neq 0$ ein $\varphi \in \operatorname{Hom}_{1}\left(\Gamma_{A}, A_{1}\right)$ mit $f h \neq 0$.

Beweis. Es genügt zu zeigen: $\mathrm{Zu}$ jedem $\xi \in T, \xi \neq 0$ gibt es ein $f \in \operatorname{Hom}_{1}\left(\Gamma_{A}, \Lambda_{A}\right)$ mit $f(\xi) \neq 0$. Nach Voraussetzung gibt es einen freien Modul

$$
F_{1}=\oplus x_{i} A=\Gamma_{A} \oplus B_{1} .
$$

$\mathrm{Zu} \xi \in \Gamma \leqq F_{A}, \xi \neq 0$ gibt es dann offenbar ein $g \in \operatorname{Hom}_{A_{1}}\left(F_{A_{1}}, \Lambda_{1 .}\right)$ mit $g(\xi) \neq 0$. Dann ist die Einschränkung $f$ von $g$ auf $\Gamma$ ein Element aus $\operatorname{Hom}_{A}\left(\Gamma_{A}, \Lambda_{A}\right)$ mit $f(\xi)=g(\xi) \neq 0$.

Hilfssatz 3: Sei $F_{A}=\bigoplus_{i=1}^{n} x_{i} \Lambda$ ein freier $\Lambda$-Rechtsmodul mit der Basis $x_{1}, \ldots, x_{n}$ und seine $f_{1}, \ldots, f_{n} \in \operatorname{Hom}_{A}\left(F_{1}, A_{A}\right)$ beliebig gegeben, dann gibt es ein $h \in \operatorname{Hom}_{A}\left(F_{.1}, F_{.1}\right)$, so daß fiir die Abbildungen $d_{i}$ aus (2)

gilt.

$$
d_{i} h=f_{i} \quad(i=1, \ldots, n)
$$

Beweis. Sei $f_{i}=\sum_{j=1}^{n} \lambda_{i j} d_{j}, \lambda_{i j} \in \Lambda$; sei ferner $h_{j} \in \operatorname{Hom}_{.1}\left(F_{A}, F_{A}\right)$ die Abbildung, die $x_{j}$ auf $\sum_{i=1}^{n} x_{i} \lambda_{i j}$ und $x_{k}(k \neq j)$ auf 0 abbildet.
Dann folgt

$$
d_{i} \sum_{j=1}^{n} h_{j}=\sum_{j=1}^{n} \lambda_{i j} d_{j}=f_{i},
$$

d.h. $h=\sum_{j=1}^{n} h_{j}$ ist die gesuchte Abbildung.

2.3. In $\operatorname{Hom}_{A}\left(\Gamma_{A}, \Gamma_{A}\right)$ ist der Ring $\Gamma^{l}$ der Linksmultiplikatoren enthalten. Wegen der Ringisomorphie $\Gamma \sim I^{l}$ kann, falls keine Verwechslung möglich ist, der Index $l$ weggelassen werden.

Satz 1: Ist $\Gamma / \Lambda$ eine Frobenius-Erweiterung bzw. cine freie Frobenius-Erweiterung, dann ist auch $\mathrm{Hom}_{1}\left(\Gamma_{A}, \Gamma_{A}\right) / \Gamma^{l}$ eine FrobeniusErweiterung bzw. eine freie Frobenius-Erweiterung.

Beweis. Wir wollen zeigen: Aus (r1) und (r 2$)$ bzw. (r 1$)$ und ( 3 ) für $\Gamma / \Lambda$ folgt (11') und (12) bzw. $\left(11^{\prime}\right)$ und (13) für $\operatorname{Hom}_{A}\left(\Gamma_{A}, \Gamma_{A}\right) / \Gamma$. Nach den Feststellungen in 1.1. folgt aus (r2) bzw. (r3) für $\Gamma / A$ zunächst (12) bzw. (13) für $\operatorname{Hom}_{A}\left(\Gamma_{A}, \Gamma_{A}\right) / T$. Es bleibt die Gültigkeit 
von (11') für $\operatorname{Hom}_{.1}\left(\Gamma_{.1}, \Gamma_{A}\right) / \Gamma$ nachzuweisen. 1)azu werden wir den nach ( $\mathrm{r} 1)$ existierenden ,rechtsseitigen" Frobenins-Isomorphismus

$$
\varphi: \operatorname{Hom}_{.1}\left(\Gamma_{.1}, A_{.1}\right) \rightarrow I
$$

$\mathrm{zu}$ einem $\left(\mathrm{l}^{\prime}\right)$ genügenden ,linksseitigen“ Frobenzus-Homomorphismus

fortsetzen.

$$
\psi: \operatorname{Hom}_{4}\left(\Gamma_{.1}, \Gamma_{.1}\right) \rightarrow \Gamma
$$

Nach Voraussetzung gibt es einen freien Modul

$$
F_{.1}=\bigoplus_{i=1}^{n} x_{i} \Lambda=\Gamma_{.1} \oplus B_{.1} .
$$

Dann folgt nach (4), daß $\varphi$ zunächst zu einem Homomorphismus $q_{1}$ von $\operatorname{Hom}_{A}\left(F_{A}, A_{A}\right)$ auf $\Gamma$ fortgesetzt werden kann. Damit ist $\phi_{1}$ für die Abbildungen $d_{i}(i=1, \ldots, n)$ erklärt. Wegen (3) existiert dann eine Fortsetzung von $\varphi_{1} z u$ einer Abbildung $\varphi_{2} \operatorname{von} \operatorname{Hom}\left(F_{A}, \Gamma_{.1}\right)$ auf $\Gamma$. Die Einschränkung $\psi$ von $\varphi_{2}$ auf $\operatorname{Hom}_{1}\left(\Gamma_{1}, \Gamma_{A}\right)$ wird dann die gewünschten Eigenschaften besitzen. Wegen $I \operatorname{Hom}_{A}\left(\Gamma_{A}, \Lambda_{A}\right)=$ $\operatorname{Hom}_{.1}\left(\Gamma_{.1}, \Gamma_{1}\right)$ kann man jedes Element aus $\operatorname{Hom}_{A}\left(\Gamma_{1}, \Gamma_{A}\right)$ als endiche Summe $\sum \gamma_{i} f_{i}$ mit $\gamma_{i} \in \Gamma, f_{i} \in \operatorname{Hom}_{.1}\left(I_{1}, A_{1}\right)$ schreiben. Nach Definition von $\psi$ gilt für $\gamma, \gamma_{1} \in \Gamma, \ell \in \operatorname{Hom}_{.1}\left(\Gamma_{1}, \Lambda_{.1}\right)$

$$
\left(\gamma_{1} f \gamma\right) \psi=\gamma_{1} \cdot \varphi(f \gamma)=\gamma_{1} \cdot \varphi(f) \cdot \gamma=\gamma_{1} \cdot(f) ; f \cdot \gamma,
$$

d.h. $\psi$ ist ein zweiseitiger $I$-Homomorphismus.

Es ist dann zu zeigen, daß

$$
\operatorname{Hom}_{A}\left(\Gamma_{A}, \Gamma_{A}\right) \exists h \rightarrow h \psi \in \operatorname{Hom}_{\Gamma}\left({ }_{r} \operatorname{Hom}_{A}\left(I_{A}, I^{\prime}\right),{ }_{\Gamma} \Gamma^{\prime}\right)
$$

ein Isomorphismus ist. Ist $h \neq 0$, so gibt es nach $H$ ilfssatz 2 ein $t \in \operatorname{Hom}_{A}\left(\Gamma_{A}, \Lambda_{A}\right)$ mit $f h \neq 0$. Dann folgt wegen $f h \operatorname{lom}_{11}\left(I_{A}, \Lambda_{A}\right)$ und da $\varphi$ ein Isomorphismus ist

$$
(f h) \psi=\varphi(f h) \neq 0 .
$$

Also ist (10) ein Monomorphismus.

Um zu zeigen, daß (10) ein Epimorphismus ist, ?enutzen wir die Gleichung

$$
\operatorname{Hom}_{A}\left(F_{A}, \Gamma_{A}\right)=\bigoplus_{i=1}^{n} \Gamma d_{i}=\operatorname{Hom}_{.1}\left(\Gamma_{.1}, \Gamma_{.1}\right) \oplus \operatorname{Hom}_{.1}\left(B_{1}, \Gamma_{A}\right) .
$$

$\psi$ wird durch die Festsetzung

$$
(f) \psi=0 \quad \text { für } \quad f \subseteq \operatorname{Hom}_{.1}\left(B_{A}, \Gamma_{.}\right)
$$


auf $\operatorname{Hom}_{.1}\left(F_{1}, T_{1}\right)$ fortgesetzt. Dann soll gezeigt werden, daß

$$
\operatorname{Hom}_{A}\left(F_{.1}, F_{.1}\right) \ni h \rightarrow h \psi \in \operatorname{Hom}_{\Gamma}\left({ }_{r} \operatorname{Hom}_{.1}\left(F_{.1}, \Gamma_{A}\right),{ }_{T} T\right)
$$

ein Epimorphismus ist. Gibt man für $d_{1}, \ldots, d_{n}$ beliebige Bilder $\gamma_{1}, \ldots, \gamma_{n} \in \Gamma$ vor, dann sei

$$
f_{i}=\varphi^{-1}\left(\gamma_{i}\right) \in \operatorname{Hom}_{A}\left(\Gamma_{A}, \Lambda_{A}\right) \leqq \operatorname{Hom}_{A}\left(F_{A}, \Lambda_{A}\right) \quad(i=1, \ldots, n) .
$$

Sei nach Hilfssatz $3 h \in \operatorname{Hom}_{A}\left(F_{1}, F_{A}\right)$ so bestimmt, daß $d_{i} h=f_{i}$ $(i=1, \ldots, n)$ gilt, dann folgt

$$
\left(d_{i} h\right) \psi=\left(f_{i}\right) \psi=\varphi\left(f_{i}\right)=\gamma_{i} \quad(i=1, \ldots, n),
$$

also ist (13) tatsächlich ein Epimorphismus.

Ist $\Gamma_{A}$ frei, d.h. $F=T$, dann ist man fertig. Im allgemeinen Fall beachte man, daß wegen (12) auch

$$
\operatorname{Hom}_{A}\left(\Gamma_{A}, F_{A}\right) \ni h \rightarrow h \psi \in \operatorname{Hom}_{\Gamma}\left({ }_{r} \operatorname{Hom}_{A}\left(F_{A}, \Gamma_{A}\right),{ }_{\Gamma} \Gamma\right)
$$

ein Epimorphismus ist. Schränkt man $h \psi$ auf $\operatorname{Hom}_{A}\left(\Gamma_{A}, \Gamma_{A}\right)$ ein, so folgt wegen (11) der behauptete Epimorphismus (10). Damit ist Satz 1 bewiesen.

2.4. Es erhebt sich natürlich die Frage, ob auch die Umkehrung von Satz 1 richtig ist. Es dürfte schwierig sein, diese Frage zu entscheiden, doch kann immerhin gezeigt werden

Satz 2: Ist $\Gamma / \Lambda$ eine Ringerweiterung und geniigt $\operatorname{Hom}_{A}\left(\Gamma_{.1}, \Gamma_{.1}\right) / \Gamma^{l}$ der Bedingung $\left(11^{\prime}\right)$, dann gibt es einen $\Lambda-\Gamma$-Monomorphismus von $\operatorname{Hom}_{A}\left(\Gamma_{A}, \Lambda_{A}\right)$ in $\Gamma$. Besitzt außerdem $\Gamma$ als $\Lambda$-Rechtsmodul einen zu $\Lambda$ isomorphen direkten Summanden, dann gibt es einen $1-\Gamma-I$ somorphismus von $\operatorname{Hom}_{A}\left(\Gamma_{A}, \Lambda_{A}\right)$ und $\Gamma$, d.h. dann ist für $\Gamma / \Lambda(\mathrm{r} 1)$ erfiullt.

Daraus und aus Satz 1 ergibt sich unmittelbar die

Folgerung: Ist $I$ als A-Rechtsmodul endlich erzengt und projektiv und besitzt $\Gamma_{A}$ einen zu $\Lambda$ isomorphen dirckten Summanden, so gilt: Dann und nur dann ist $\Gamma / \Lambda$ Frobenius-Erweiterung, wenn $\operatorname{Hom}_{.1}\left(\Gamma_{.1}, \Gamma_{1}\right) / \Gamma^{l}$ Frobenius-Erweiterung ist.

Insbesondere sind die Voraussetzungen der Folgerung erfüllt, wenn $\Gamma_{.1}$ endlich erzeugt und frei ist. Dann ergibt sich das anfangs erwähnte Resultat.

Beweis von Satz 2. Nach Voraussetzung gibt es einen (11') genügenden Homomorphismus $\psi$ von $\operatorname{Hom}_{1}\left(\Gamma_{A}, \Gamma_{A}\right)$ in $\Gamma$. Die Einschränkung von $\psi$ auf $\operatorname{Hom}_{.1}\left(\Gamma_{.1}, A_{.1}\right)$ sei $\varphi$, wobei für $f \in \operatorname{Hom}_{.1}\left(\Gamma_{.1}, A_{.1}\right)$ 
$\varphi(f)=(f) \psi$ geschrieben wird. Da $\psi$ ein zweiseitiger $l$-Homomorphismus ist, ist jedenfalls $\varphi$ ein $\Lambda-\Gamma$-Homomorphismus. Sei nun für $f \in \operatorname{Hom}_{.1}\left(\Gamma_{A}, A_{.1}\right) \quad \varphi(f)=(f) \psi=0$, dann folgt $(\Gamma f) \psi=0$ und wegen Hilfssatz 1 sogar $\left(\operatorname{Hom}_{A}\left(I_{A}, \Gamma_{A}\right) f\right) \psi=0$, also gilt wegen $\left(11^{\prime}\right) f=0$, d.h. $\varphi$ ist ein Monomorphismus.

Sei nun

$$
\Gamma=x \Lambda \oplus A_{A}, \quad x \Lambda_{A} \simeq \Lambda_{A},
$$

und sei $\gamma \in \Gamma$ beliebig gegeben. Wir definieren $d \in \operatorname{Hom}_{A}\left(\Gamma_{A}, A_{A}\right)$ durch die Festsetzung

dann folgt

$$
d(x)=1, \quad d(A)=0,
$$

$$
\operatorname{Hom}_{.1}\left(\Gamma_{.1}, \Gamma_{A}\right)=\Gamma d \oplus \operatorname{Hom}_{.1}\left(A_{.1}, \Gamma_{A}\right) .
$$

Sei nun $\sigma \in \operatorname{Hom}_{\Gamma}\left({ }_{\Gamma} \operatorname{Hom}_{.1}\left(\Gamma_{A}, \Gamma_{A}\right),{ }_{\Gamma} \Gamma\right)$ definiert durch

$$
\text { (d) } \sigma=\gamma,\left(\operatorname{Hom}_{A}\left(A_{A}, \Gamma_{A}\right)\right) \sigma=0,
$$

dann gibt es nach Voraussetzung ein $h \in \operatorname{Hom}_{.1}\left(\Gamma_{.1}, \Gamma_{.1}\right)$ mit $\sigma=h \psi$. Es folgt

$$
\gamma=(d) \sigma=(d h) \psi=\varphi(d h) .
$$

Somit ist $\varphi$ ein Epimorphismus.

Es bleibt die Frage, ob man die Voraussetzung, daß $\Gamma_{.1}$ einen zu 1 isomorphen direkten Summanden besitzt, vermeiden kann.

\section{Homologische Eigenschaften von Frobenius-Erweiterungen}

\subsection{Allgemeine Eigenschaften}

Wir erinnern zunächst an einige Begriffe und Resultate aus der relativen homologischen Algebra (relativ in bezug auf ein Ringpaar). Sei wie bisher $T$ ein Ring mit 1-Element und $A$ ein Lnterring mit dem gleichen 1-Element. Eine Folge von $l$-Rechtsmoduln und l'-Homomorphismen

$$
\cdots \rightarrow A_{i+1} \stackrel{\alpha_{i+1}}{\rightarrow} A_{i} \stackrel{\alpha_{i}}{\rightarrow} A_{i-1} \cdots \cdots
$$

heißt $(I, A)$-exakt, wenn sie $I^{\prime}$-exalkt ist und für jedes $i \operatorname{Ke}\left(\alpha_{i}\right)$ und $\operatorname{Bi}\left(\alpha_{i}\right)$ 1-direkte Summanden in $A_{i}$ bzw. $A_{i-1}$ sind. Der $\Gamma$-Rechtsmodul $B$ heißt $(I, A)$-projektiv bzw. $(I, A)$-injektiv, wenn für jede $(I, A)$-exakte Folge $(14)$ auch die Folge

$$
\begin{aligned}
\cdots \rightarrow \operatorname{Hun}_{I^{\prime}}\left(B, A_{i+1}\right) & \stackrel{\operatorname{Hom}\left(1, \alpha_{i+1}\right)}{\longrightarrow} \\
\stackrel{\operatorname{Hom}\left(1, \alpha_{i j}\right)}{\longrightarrow} & \operatorname{Hom}_{\Gamma}\left(B, A_{i}\right) \\
& \operatorname{Hom}_{\Gamma}\left(B, A_{i-1}\right) \rightarrow \cdots
\end{aligned}
$$


bzw.

$$
\begin{aligned}
& \cdots \rightarrow \operatorname{Hom}_{\Gamma}\left(A_{i-1}, B\right) \stackrel{\operatorname{Hom}\left(x_{i}, 1\right)}{\longrightarrow} \operatorname{Hom}_{\Gamma}\left(A_{i}, B\right) \\
& \stackrel{\operatorname{Hom}\left(x_{i+1}, 1\right.}{\longrightarrow} \operatorname{Hom}_{I}\left(A_{i \div 1}, B\right) \rightarrow \cdots
\end{aligned}
$$

exakt ist. Es gelten dann folgende Aussagen ${ }^{1}$ :

(P1) Ist $C$ ein beliebiger $A$-Rechtsmodul, dann ist $C Q_{A}$ ein $(I, A)$-projektiver $\Gamma$-Rechtsmodul.

(P2) Ist $C$ ein projektiver $\Lambda$-Rechtsmodul, dann ist $C Q_{1} \Gamma$ ein projektiver $T$-Rechtsmodul.

(P3) Für einen $\Gamma$-Rechtsmodul $C$ sind folgende Eigenschaften äquivalent:

(a) $C$ ist $(I, \Lambda)$-projektiv;

(b) Jede $(I, A)$-exakte Folge $B \rightarrow C \rightarrow 0$ ist $(I, I)$-exakt;

(c) $C \otimes I$ besitzt einen zu $C$ isomorphen $T$-direkten Summanden.

(I1) Ist $C$ ein beliebiger $A$-Rechtsmodul, dann ist $\operatorname{Hom}_{.1}(I, C)$ ein $(T, \Lambda)$-injektiver $\Gamma$-Rechtsmodul.

(I2) Ist $C$ ein injektiver $A$-Rechtsmodul, dann ist $\operatorname{Hom}_{11}(I, C)$ ein injektiver $\Gamma$-Rechtsmodul.

(I3) Für einen $\Gamma$-Rechtsmodul $C$ sind folgende Eigenschaften äquivalent:

(a) $C$ ist $(I, A)$-injektiv;

(b) Jede $(\Gamma, \Lambda)$-exakte Folge $0 \rightarrow C \rightarrow B$ ist $(\Gamma, \Gamma)$-exakt;

(c) $\operatorname{Hom}_{A}(I, C)$ besitzt einen zu $C$ isomorphen $I$-direkten Summanden.

Daraus ergeben sich für eine Frobenius-Erweiterung $I / \Lambda$ sofort eine Reihe von Folgerungen.

(I) Ist $\Lambda$ rechtsseitig selbstinjektiv, dann auch $\Gamma$. Speziell: Eine Frobenius-Erweiterung eines Quasi-Frobenius-Rings ist ein QuasiFrobenius-Ring ${ }^{2}$.

Beweis: Die erste Behauptung folgt aus (r1) und (I2). Die Quasi-Frobenius-Ringe sind genau die rechtsseitig selbstinjektiven

${ }^{1}$ Siehe [8] oder [2]; $(\Gamma, \Lambda)$-projektive bzw. $(\Gamma, \Lambda)$-injektive Moduln werden in 2] $\varphi$-projektiv bzw. $\varphi$-injektiv genannt. In [9], wo diese Begriffe zum erstenmal in der Literatur für beliebige Ringerweiterungen $\Gamma / \Lambda$ auftreten, werden sie im Anschluß an [4] als $M_{0}$ - bzw. $M_{u}$-Moduln bezeichnet.

2 Spezialfälle s. [9], Satz 10; [3], Corollary 9, Corollary 20; [7], Corollary 4, Theorem 6. 
Ringe mit Minimalbedingung für Rechtsideale [3]. Daraus folgt die zweite Behauptung.

(II) Für einen beliebigen $\Lambda$-Rechtsmodul $C$ gilt

$$
C \otimes \Gamma \cong \operatorname{Hom}_{\Lambda}(\Gamma, C) \text {. }
$$

Beweis: Folgt aus (6) und (11).

(III) Ein $\Gamma$-Rechts-(oder Linksmodul) ist dann urd nur dann $(\Gamma, \Lambda)$-projektiv, wenn er $(\Gamma, \Lambda)$-injektiv ist.

Beweis: Folgt aus (P3), (I3) und (II).

Wir bezeichnen die projektive bzw. injektive bzw. schwache Dimension des $T$-Moduls $C$ mit p-dim ${ }_{\Gamma}(C)$ bzw. i-dim ${ }_{\Gamma}(C)$ bzw. s- $\operatorname{dim}_{\Gamma}(C)$. Die entsprechenden $(I, \Lambda)$-Dimensionen von $C$ werden mit p-dim $\operatorname{di,\Lambda )}_{(C)}\left(C z w . \mathrm{i}-\operatorname{dim}_{(\Gamma, \Lambda)}(C)\right.$ bzw. s- $\operatorname{dim}_{(\Gamma, \Lambda)}(C)$ bezeichnet. Für die globale Dimension des Ringes $\Gamma$ bzw. der Ringerweiterung $\Gamma / \Lambda$ schreiben wir g-dim $(\Gamma)$ bzw. g-dim $(\Gamma, \Lambda)$.

(IV) Für einen beliebigen $\Gamma$-Rechtsmodul $C$ gilt $^{3}$

$$
\mathrm{p}-\operatorname{dim}_{(\Gamma, \Lambda)}(C)=\mathrm{i}-\operatorname{dim}_{(\Gamma, \Lambda)}(C)=\left\{\begin{array}{l}
0 \\
\infty
\end{array} .\right.
$$

Beweis: Wegen (III) sowie (P3) und (I3) kann man jede endliche $(\Gamma, \Lambda)$-projektive bzw. $(\Gamma, \Lambda)$-injektive Auflösung zla einer der Länge 0 verkürzen. Wegen (III) folgt die Behauptung.

(V) Jeder $\Gamma$-Rechtsmodul besitzt eine $(\Gamma, \Lambda)$-vollständige Auflösung.

Beweis: Klar wegen (III). gilt $^{4}$

(VI) Für einen beliebigen $\Gamma$-Rechtsmodul $A$ und $\Lambda$-Rechtsmodul C

$$
\operatorname{Ext}_{(\Gamma, \Lambda)}^{i}(A, C \underset{\Lambda}{\otimes} \Gamma)=\operatorname{Ext}_{(r, \Lambda)}^{i}\left(\operatorname{Hom}_{\Lambda}(I, C), A\right)=0, \quad i>0 .
$$

Beweis: Wegën (II), (P1) und (I 1) ist $C \otimes \Gamma(\Gamma, \Lambda)$-injektiv und $\operatorname{Hom}_{\Lambda}(\Gamma, C)(\Gamma, \Lambda)$-projektiv, woraus die Behauptung folgt.

(VII) Für beliebige Modwn $A_{\Lambda},{ }_{\Gamma} C$ bzw. $A_{\Gamma},{ }_{1} C$ gilt bzw. $\left.\quad \begin{array}{l}\operatorname{Tor}_{i}^{A}(A, C) \cong \operatorname{Tor}_{i}^{\Gamma}\left(\operatorname{Hom}_{\Lambda}(\Gamma, A), C\right) \\ \operatorname{Tor}_{i}^{A}(A, C) \cong \operatorname{Tor}_{i}^{\Gamma}\left(A, \operatorname{Hom}_{\Lambda}(\Gamma, C)\right)\end{array}\right\} \quad(i=0,1, \ldots)$.

${ }^{3}$ Vgl. [5], Prop. 1-3.

4 Spezialfall in [7], Prop. 7. 
Fïr beliebige Moduln $A_{.1}, C_{1}$ bzw. $A_{1}, C_{A}$ gilt $^{5}$

bzw.

$$
\left.\begin{array}{l}
\operatorname{Ext}_{.1}^{i}(A, C) \simeq \operatorname{Ext}_{\Gamma}^{i}\left(\operatorname{Hom}_{A}(T, A), C\right) \\
\operatorname{Ext}_{.1}^{i}(A, C) \simeq \operatorname{Ext}_{\Gamma}^{i}(A, C \otimes \Gamma) .
\end{array}\right\} \quad(i=0,1, \ldots) .
$$

Beweis: Folgt wegen (r2), (12) und (II) aus [2], S. 116-118. case $1-4$.

Aus (VII) folgt unmittelbar

(VIII) Fïr einen beliebigen A-Rechtsmodul A gilt:

$$
\begin{aligned}
\mathrm{s}-\operatorname{dim}_{\Gamma}\left(\operatorname{Hom}_{A}(I, A)\right) & \leqq \mathrm{s}-\operatorname{dim}_{A}(A) . \\
\mathrm{p}-\operatorname{dim}_{\Gamma}\left(\operatorname{Hom}_{A}(T, A)\right) & \leqq \mathrm{p}-\operatorname{dim}_{A}(A) . \\
\mathrm{i}-\operatorname{dim}_{\Gamma}(A \otimes \Gamma) & \leqq \mathrm{i}-\operatorname{dim}_{A}(A) .
\end{aligned}
$$

Für die nächsten Folgerungen brauchen wir einen Hilfssatz, bei dem nicht vorausgesetzt wird, daß $\Gamma / \Lambda$ Frobenius-Erweiterung ist.

Hilfssatz 4: Sei $\Gamma$ ein Ring mit 1-Element und $A$ ein Unterring mit dem gleichen 1-Element.

a) Ist $\Gamma_{.1}$ projektiver $A-M o d u l$, dann istjeder I'-projektive Modul $A_{\Gamma}$, auch als 1-Modul projektiv.

b) Ist ${ }_{1} \Gamma$ projektiver 1 -Modul, dann ist jeder $\Gamma$-injektive Modul $A_{\Gamma}$ auch as 1 -Modul injektiv.

Beweis $^{6}$ : a) Die Behauptung folgt aus der Tatsache, daß ein projektiver Modul direkter Summand eines freien Moduls ist und da $B$ direkte Summen und direkte Summanden von projektiven Moduln wieder projektiv sind.

b) Als $\Gamma$-injektiver Modul ist $A_{\Gamma}$ (bis auf Isomorphie) direkter Summand eines injektiven Moduls der Form $\operatorname{Hom}_{Z}(I, T)$, wobei $T$ eine teilbare Abelsche Gruppe und $Z$ der Ring der ganzen Zahlen ist. Da ${ }_{1} I$ ' projektiv ist, gibt es einen freien $\Lambda$-Modul ${ }_{1} F$ mit

$$
{ }_{1} F={ }_{1} \Gamma \oplus{ }_{1} B=\oplus_{i} \Lambda x_{i}, \quad{ }_{1} A x_{i} \sim{ }_{1} \Lambda \text {. }
$$

Dann folgt

$$
\operatorname{Hom}_{Z}(F, T) \simeq \operatorname{Hom}_{Z}(I, T) \oplus \operatorname{Hom}_{Z}(B, T)
$$

5 Spezialfälle in 3], Prop. 7; 7]; Prop. 2.

${ }^{6}$ Der Beweis von a) ist wohlbekannt. $\mathrm{Zu} \mathrm{b}$ ) siehe $[2]$, S. 31; der Beweis wird dort aber nicht ausgeführt; außerdem fehlt clort Angabe der Seite, für die $I$ ' als $A$-Modul projektiv sein muß. Vgl. auch [2], S. 123, Ex. 10. Wir führen den Beweis clual zu dem bekannten Beweis von a), den wir kurz. angeben. 
als 1 -Rechtsmoduln. Andererseits gilt

$$
\operatorname{Hom}_{z}(F, T)=\operatorname{Hom}_{Z}\left(\oplus_{i} A x_{i}, T\right) \sim I_{i} I \operatorname{Hom}_{z}\left(1 x_{i}, T\right),
$$

und somit ist $\operatorname{Hom}_{Z}(F, T)$ als direktes Produkt der injektiven 1-Rechtsmoduln $\operatorname{Hom}_{Z}\left(A x_{i}, T\right) \simeq \operatorname{Hom}_{Z}(1, T)$ wieder injektiv und dann auch $A$ als direkter Summand.

Sei jetzt wieder $\Gamma / \Lambda$ eine Frobenius-Erweiterung.

(IX) Für cinen $\Gamma$-Rechtsmodul A endlicher schwacher bzu. projektiver bzw. injektiver Dimension gilt '

$$
\mathrm{s}-\operatorname{dim}_{\Gamma}(A) \leqq \mathrm{s}-\operatorname{dim}_{.1}(A)
$$

$b z w$.

$b z w$.

$$
\mathrm{p}-\operatorname{dim}_{I}(A)=\mathrm{p}-\operatorname{dim}_{.1}(A)
$$

$$
\mathrm{i}-\operatorname{dim}_{\Gamma}(A)=\mathrm{i}-\operatorname{dim}_{.1}(-A) \text {. }
$$

Beweis: Wir beweisen nur die dritte Behauptung und schreiben $C$ statt $A$. Sei $\mathrm{i}-\operatorname{dim}_{\Gamma}(C)=n<\infty$ und sei $A_{\Gamma}$. mit $\operatorname{Ext}_{\Gamma}^{n}(A, C) \neq 0$. Die Folge der $T$-Rechtsmoduln

$$
0 \rightarrow A \rightarrow \operatorname{Hom}_{.1}(I, A) \rightarrow B \rightarrow 0
$$

mit $B=\operatorname{Hom}_{A 1}(I, A) / \operatorname{Hom}_{\Gamma}(I, A)$ ist exakt. Dann gilt wegen $\operatorname{Ext}_{\Gamma}^{n+1}(B, C)=0$, daß

$$
\operatorname{Ext}_{\Gamma}^{n}\left(\operatorname{Hom}_{.1}(I, A), C\right) \rightarrow \operatorname{Ext}_{\Gamma}^{n}(A, C)
$$

ein Epimorphismus ist. Wegen (VII) gilt folglich $\operatorname{Ext}_{.1}^{\prime \prime}(A, C) \neq 0$, also $\mathrm{i}-\operatorname{dim}_{\Gamma^{\prime}}(C) \leqq \mathrm{i}-\operatorname{dim}_{.1}(C)$. Die Umkehrung ist klar, da nach dem Hilfssatz jede injektive Auflösung von $C_{r}$ auch eine solche von $C_{\mathrm{I}}$. ist.

Wir brauchen nun einen weiteren Hilfssatz, bei dem wir nicht voraussetzen, daß $\Gamma / \Lambda$ Frobenius-Erweiterung ist.

Hilfssatz 58: Sei $\Gamma$ cin Ring mit 1-Elemenl und de in Unterring mit dem gleichen 1-Element. Dann gilt

a) Ist $A_{\Gamma^{\prime}}(\Gamma, A)$-projektiv und ist ${ }_{1} I$ - 1 -projcktim, tann gilt fiur beliebigen Moduln $C_{\Gamma}$ :

$\operatorname{Ext}_{\Gamma}^{i}(A, C)$ ist zu einem direkten Summanden im $\mathrm{Ext}_{A}^{i}(A, C)$ isomorph.

b) Ist $C_{\Gamma}(\Gamma, \Lambda)$-injektiv und ist $\Gamma_{.1} A$-projcktir, dann gilt fiur beliebigen Modui $A_{I}$ :

7 Vgl. [3], Theorem 10; 7), Theorem 5.

8 Vgl. [8], Prop. 1.

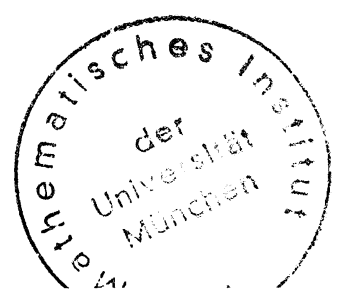


$\operatorname{Ext}_{T}^{i}(A, C)$ ist zu einem direkten Summanden von $\operatorname{Ext}_{.1}^{i}(A, C)$ isomorph.

c) Ist $A_{\Gamma}(I, A)$-projektiv und ${ }_{1} I$ A-projektiv, dann gilt für beliebigen Modul ${ }_{\Gamma} C$ :

$\operatorname{Tor}_{i}^{\Gamma}(A, C)$ ist zu einem direkten Summanden von $\operatorname{Tor}_{i}^{-1}(A, C)$ isomorph.

d) Ist ${ }_{\Gamma} C(I, \Lambda)$-projektiv und $\Gamma_{A} A$-projektiv, dann gilt fiir beliebigen Modul $A_{\Gamma}$ :

$\operatorname{Tor}_{i}^{T}(A, C)$ ist zu einem direkten Summanden von $\operatorname{Tor}_{i}^{1}(A, C)$ isomorph.

Beweis: Wir beweisen nur a), da die anderen Behauptungen analog zu zeigen sind. $\mathrm{Da} A(\Gamma, A)$-projektiv ist, ist $A \mathrm{zu}$ einem $I$-direkten Summanden von $A \underset{A}{\otimes} \Gamma$ isomorph. Wegen $\operatorname{Hom}_{\Gamma}(A \otimes \Gamma, C) \simeq \operatorname{Hom}_{1}(A, C) \quad($ siehe $[2]$, S. 118) ist dann $\operatorname{Hom}_{\Gamma}(A, C)$ zu einem direkten Summanden von $\operatorname{Hom}_{A 1}(A, C)$ isomorph. Da ${ }_{1} \Gamma \Lambda$-projektiv ist, ist nach Hilfssatz 4 jede $\Gamma$-injektive Auflösung von $C$ auch eine 4 -injektive Auflösung von $C$. Daraus erhält man die Behauptung.

Für eine Frobenius-Erweiterung $\Gamma / \Lambda$ ergibt sich aus diesem Hilfssatz unmittelbar:

(X) Ist einer der Moduln $A_{\Gamma}, C_{\Gamma}(\Gamma, \Lambda)$-projektiv $(=(I, A)$ injektiv), dann ist $\operatorname{Ext}_{\Gamma}^{i}(A, C)$ zu einem direktein Summanden von $\operatorname{Ext}_{A}^{i}(A, C)$ isomorph ${ }^{9}$. Ist einer der Moduln $A_{l},{ }_{\Gamma} C(I, \Lambda)$-projektiv $(=(I, A)$-injektiv $)$, dann ist $\operatorname{Tor}_{i}^{\Gamma}(A, C)$ zu einem direkten Summanden von $\operatorname{Tor}_{i}^{A}(A, C)$ isomorph.

Aus den Hilfssätzen 4 und 5 folgt schließlich

(XI) Ist $A(\Gamma, \Lambda)$-projektiv, dann gilt

$$
\begin{aligned}
\mathrm{s}-\operatorname{dim}_{\Gamma}(A) & \leqq \mathrm{s}-\operatorname{dim}_{A}(A) \\
\mathrm{p}-\operatorname{dim}_{\Gamma}(A) & =\mathrm{p}-\operatorname{dim}_{.1}(A) \\
\mathrm{i}-\operatorname{dim}_{\Gamma}(A) & =\mathrm{i}-\operatorname{dim}_{A}(A) .
\end{aligned}
$$

\subsection{Ausgezeichnete Frobenius-Erweiterungen}

Wir wollen eine Ringerweiterung $\Gamma / \Lambda$ ausgezeichnet nennen, wenn $\Gamma$ als zweiseitiger $\Lambda$-Modul einen zu $\Lambda$ isomorphen direkten Summanden besitzt.

${ }^{9}$ Spezialfall $\Gamma=$ Gruppenring mit Koeffizienten aus kommutativem Ring $A$ siehe [3], Prop. 14; vgl. auch [5], Prop. 4. 
Beispiele für ausgezeichnete Frobenius-Erweiterungen kann man sofort angeben. Sei (5) eine beliebige Gruppe, in eine Untergruppe von endlichem Index in (5), und sei $R$ ein Ring mit 1-Element, dann ist der Gruppenring $R[(S)$ ausgezeichnete (freic) Frobenius-Erweiterung von $R[\check{i}]$.

Eine ausgezeichnete Frobenius-Erweiterung hat man auch in dem Falle, daß $\Gamma .1$ eine freie Frobenius-Erweiterung und $A$ im Zentrum von $\Gamma$ enthalten ist. Sind nämlich $r_{1}, \ldots, r_{n}$ und $l_{1}, \ldots, l_{n}$ duale Basen und ist $\psi$ der Frobenius-Homomorphismus mit $\psi\left(l_{i} r_{j}\right)=\delta_{i j}$, dann hat man die Zerlegung $\Gamma=l_{1} r_{1} \mathcal{A} \oplus \operatorname{Ke}(\psi)$ in zweiseitige $A$-Moduln, wobei $l_{1} r_{1} A$ als zweiseitiger $A$-Modul zu $A$ isomorph ist.

Diese Beispiele zeigen die Bedeutung der ausgezeichneten Frobenius-Erweiterungen.

Hilfssatz 6: a) Ist $\Gamma / \Lambda$ eine ausgezeichnete Ringerweiterung, sind ${ }_{A} \Gamma$ und $\Gamma_{1}$ projektive $A$-Moduln und sind $A, C$ beliebige 1-Rechtsmoduln, dann ist $\operatorname{Ext}_{.1}^{i}(A, C)$ isomorph zu einem direkten Summanden von $\operatorname{Ext}_{\Gamma}^{i}(A \otimes \Gamma, C \otimes \Gamma), \operatorname{Ext}_{\Gamma}^{i}\left(A \otimes_{1} \Gamma, \operatorname{Hom}_{A}(T, C)\right)$, $\operatorname{Ext}_{\Gamma}^{i}\left(\operatorname{Hom}_{.1}(\Gamma, A), C \otimes \Gamma\right) \stackrel{.1}{\otimes}$ und $\operatorname{Ext}_{\Gamma}^{i}\left(\operatorname{Hom}_{A}(T, A), \operatorname{Hom}_{.1}(T, C)\right)$.

b) Voraussetzungen über $\Gamma / \Lambda$ wie in a). Sind A cin A-Rechts-und $C$ ein $A$-Linksmodul, dann ist $\operatorname{Tor}_{i}^{\Lambda}(A, C)$ isomorph zu einem direkten Summanden von $\operatorname{Tor}_{i}^{\Gamma}(A \otimes \Gamma, C \otimes \Gamma), \operatorname{Tor}_{i}^{\Gamma}\left(A \otimes \Gamma, \operatorname{Hom}_{.1}(\Gamma, C)\right)$, $\operatorname{Tor}_{i}^{\Gamma}\left(\operatorname{Hom}_{A}(\Gamma, A), C \otimes \Gamma\right) \stackrel{A}{A}$ und $\operatorname{Tor}_{i}^{T}\left(\operatorname{Hom}_{A}(\Gamma, A), \operatorname{Hom}_{A}(\Gamma, C)\right)$.

Beweis $^{10}$ : Da die Beweisführung in allen Fällen analog verläuft, können wir uns darauf beschränken zu zeigen, dal $\operatorname{Ext}_{A}^{i}(A, C)$ $z u$ einem direkten Summanden von $\operatorname{Ext}_{\Gamma}^{i}\left(A_{.1}^{\otimes} I, M \otimes \Gamma\right)$ isomorph ist. Sei $I=\Lambda^{\prime} \oplus \Lambda^{\prime \prime}$ die nach Voraussetzung existierende Zerlegung von $\Gamma$ in zweiseitige $A$-Moduln mit $A^{\prime} \sim A$. Dann folgt

$$
\begin{aligned}
\operatorname{Ext}_{.1}^{i}(A, M \otimes \Gamma) & \simeq \operatorname{Ext}_{.1}^{i}\left(A, M \otimes A^{\prime}\right) \oplus \operatorname{Ext}_{A}^{i}\left(A, M \otimes A^{\prime \prime}\right) \\
& \simeq \operatorname{Ext}_{.1}^{i}(A, M) \oplus \operatorname{Ext}_{.1}^{i}\left(A, M A^{\prime \prime}\right) .
\end{aligned}
$$

Da ${ }_{4} \Gamma$ projektiv ist, folgt (nach $[2]$, S. 118)

$$
\operatorname{Ext}_{A}^{i}(A, M \otimes \Gamma) \simeq \operatorname{Ext}_{I}^{i}\left(A \otimes \Gamma, M Q_{.1} \Gamma\right)
$$

womit der Beweis geführt ist.

${ }^{10}$ Vgl. [5], Beweis von Prop. 6. 
Aus diesem Hilfssatz entnimmt man unmittelbar die

Folgerung: Sei $\Gamma / \Lambda$ ausgezeichnet, seien ${ }_{A} \Gamma$ und $\Gamma_{A}$ projektiv, und sei $A$ ein beliebiger $A$-Rechtsmodul, dann gilt ${ }^{11}$

$$
\begin{aligned}
\mathrm{p}-\operatorname{dim}_{A}(A) & \leqq \mathrm{p}-\operatorname{dim}_{\Gamma}(A \otimes \Gamma), \\
\mathrm{p}-\operatorname{dim}_{A}(A) & \leqq \mathrm{p}-\operatorname{dim}_{\Gamma}\left(\operatorname{Hom}_{A}(I, A)\right), \\
\mathrm{i}-\operatorname{dim}_{A}(A) & \leqq \mathrm{i}-\operatorname{dim}_{\Gamma}(A \otimes \Gamma), \\
\mathrm{i}-\operatorname{dim}_{A}(A) & \leqq \mathrm{i}-\operatorname{dim}_{\Gamma}\left(\operatorname{Hom}_{A}(I, A)\right), \\
\mathrm{s}-\operatorname{dim}_{A}(A) & \leqq \mathrm{s}-\operatorname{dim}_{\Gamma}(A \otimes \Gamma), \\
\mathrm{s}-\operatorname{dim}_{A}(A) & \leqq \mathrm{s}-\operatorname{dim}_{\Gamma}\left(\operatorname{Hom}_{A}(I, A)\right), \\
\mathrm{g}-\operatorname{dim}(A) & \leqq \mathrm{g}-\operatorname{dim}(\Gamma) .
\end{aligned}
$$

Wendet man diese Abschätzung auf eine ausgezeichnete FrobeniusErweiterung an, so erhält man unter Beachtung von (VIII) den

Satz $3^{12}$ : Ist $\Gamma / \Lambda$ eine ausgezeichnete Frobenius-Erweiterung, dann gilt fïr jeden A-Rechtsmodul $A$ :

$$
\begin{aligned}
& \mathrm{s}-\operatorname{dim}_{\Gamma}(A \otimes \Gamma)=\mathrm{s}-\operatorname{dim}_{\Gamma}\left(\operatorname{Hom}_{\Lambda}(\Gamma, A)\right)=\mathrm{s}-\operatorname{dim}_{A}(A), \\
& \mathrm{p}-\operatorname{dim}_{\Gamma}(A \underset{A}{\otimes} \Gamma)=\mathrm{p}-\operatorname{dim}_{\Gamma}\left(\operatorname{Hom}_{A}(T, A)\right)=\mathrm{p}-\operatorname{dim}_{A}(A), \\
& \mathrm{i}-\operatorname{dim}_{\Gamma}(A \underset{A}{\otimes} \Gamma)=\mathrm{i}-\operatorname{dim}_{\Gamma}\left(\operatorname{Hom}_{A}(T, A)\right)=\mathrm{i}-\operatorname{dim}_{A}(A) .
\end{aligned}
$$

Wir weisen schließlich darauf hin, daß man die zuvor angegebene Folgerung auch mit (IX) und (XI) zu neuen Dimensionsgleichungen verknüpfen kann.

\subsection{Eigenschaften der Spur}

Wir wollen uns jetzt auf freie Frobenius-Erweiterungen beschränken. Dann gibt es, wie in 1.4. ausgeführt, eine Rechtsbasis $r_{1}, \ldots, r_{n}$ und eine Linksbasis $l_{1}, \ldots, l_{n}$ von $\Gamma / \Lambda$, die zueinander dual sind. Seien $M$ und $N$ beliebige $\Gamma$-Rechtsmoduln und sei $g \in \operatorname{Hom}_{.1}(M, N)$, dann betrachtet man die aus der Kohomologie der Gruppen bekannte Abbildung

$$
\operatorname{Spur}(g)=\sum_{i=1}^{n} l_{i}^{*} g r_{i}^{\prime},
$$

wobei $r_{i}^{\prime}$ bzw. $l_{i}^{*}$ der durch $r_{i}$ bzw. $l_{i}$ erzeugte Rechtsmultiplikator von $M$ bzw. $N$ ist.

${ }^{11}$ Die globale Dimensionsabschätzung enthält [5], Prop. 6.

12 Enthält [3], Corollary $S^{\prime}$; [5], Prop. 7. 
Die Spur besitzt die beiden folgenden Eigenschaften, von denen wir allein Gebrauch machen.

1. Aus $g \in \operatorname{Hom}_{A}(M, N)$ folgt $\operatorname{Spur}(g) \in \operatorname{Hom}_{1}(M, N)$.

2. Sind $L$ und $Q$ weitere $\Gamma$-Rechtsmoduln und gilt

$$
g \in \operatorname{Hom}_{.1}(M, N), \quad t_{1} \in \operatorname{Hom}_{\Gamma}(N, Q), \quad f_{2} \in \operatorname{Hom}_{\Gamma}(L, M),
$$

dann folgt $\operatorname{Spur}\left(t_{1} g f_{2}\right)=f_{1} \operatorname{Spur}(g) f_{2}$.

Die erste Eigenschaft folgt aus der Dualität der Basen; die zweite ist unmittelbar klar.

Sei jetzt $f \in \operatorname{Hom}_{\Gamma}(M, N)$, sei $A$ ein $\Gamma$-Rechtsmodul und bezeichnet $1_{A}$ die identische Abbildung von $A$, dann induziert $f$ in bekannter Weise einen Homomorphismus $\operatorname{Ext}_{(T, A)}^{i}\left(1_{A}, f\right)$ von $\operatorname{Ext}_{(T, \Lambda)}^{i}(A, M)$ in $\operatorname{Ext}_{(T, A)}^{i}(A, N)$. Die entsprechende Bemerkung gilt auch für das erste Argument, wobei nur die Kontravarianz zu beachten ist.

\section{Satz $4^{13}$ :}

a) Seien $A, M, N$ beliebige $\Gamma$-Rechtsmoduln und seig $\in \operatorname{Hom}_{A}(M, N)$, dann gilt

$$
\operatorname{Ext}_{(\Gamma, A)}^{i}\left(1_{A}, \operatorname{Spur}(g)\right)=0, \quad i=1,2, \ldots .
$$

b) Seien $A, B, N$ beliebige $\Gamma$-Rechtsmoduln und sei $g \in \operatorname{Hom}_{A}(B, A)$, dann gilt

$$
\operatorname{Ext}_{(\Gamma, 1)}^{i}\left(\operatorname{Spur}(g), 1_{M}\right)=0, \quad i=1,2, \ldots
$$

Bemerkung: Die analoge Aussage gilt auch für $\operatorname{Tor}_{i}^{(\Gamma, A)}$.

Beweis: Wir beweisen nur a). Sei

$$
\cdots \stackrel{\alpha_{3}}{\rightarrow} A_{2} \stackrel{\alpha_{2}}{\rightarrow} A_{1} \stackrel{\alpha_{1}}{\rightarrow} A_{0} \rightarrow A \rightarrow 0
$$

eine $(\Gamma, A)$-projektive Auflösung von $A$. Dann gilt es

mit

$$
h_{i} \in \operatorname{Hom}\left(A_{i}, A_{i+1}\right)
$$

$$
1_{A_{i}}=h_{i-1} \alpha_{i}+\varkappa_{i \div 1} h_{i} .
$$

Ist $f \in \operatorname{Hom}_{\Gamma}\left(A_{i}, M\right)$ und $f \in \operatorname{Ke}\left(\operatorname{Hom}\left(\alpha_{i+1}, 1_{M}\right)\right)$, (l.h. $f \alpha_{i+1}=0$, dann folgt

Daraus erhält man

$$
f=f 1_{A_{i}}=f h_{i-1} x_{i} .
$$

$$
\operatorname{Spur}(g) f=\operatorname{Spur}(g f)=\operatorname{Spur}\left(g f h_{i-1} \alpha_{i}\right)=\operatorname{Spur}\left(g f h_{i-1}\right) \alpha_{i},
$$

${ }_{13}$ Dieser Satz stellt eire Verallgcmeincrung vor Salz $1 \mathrm{i}$ als [i] dar, wo $\Gamma$ ein Gruppenring ist. Der Beweis kann aus [1] übernommen werden, doch wollen wir ihn der Vollständigkeit halber angeben. 
also

$$
\operatorname{Spur}(g) t \in \operatorname{Bi}\left(\operatorname{Hom}\left(\alpha_{i}, 1_{N}\right)\right)
$$

und somit gilt wie behauptet

$$
\operatorname{Ext}_{(\Gamma, A)}^{i}\left(1_{A}, \operatorname{Spur}(g)\right)=0 .
$$

Bezeichne $Z_{\Gamma}(\Lambda)$ den Zentralisator von $\Lambda$ in $\Gamma$, dann ist für jeden $\Gamma$-Rechtsmodul die Multiplikation einem $\tau \in Z_{\Gamma}(\Lambda)$ ein $\Lambda$-Endomorphismus und $\operatorname{Spur}\left(Z_{\Gamma}(\Lambda)\right)=\sum_{i=1}^{n} r_{i} Z_{\Gamma}(\Lambda) l_{i}$ ist ein Ideal des Zentrums von $\Gamma$. Aus dem Satz folgt, daß dieses Ideal jeden Modul $\operatorname{Ext}_{(T, A)}^{i}(A, M) \quad(i=1,2, \ldots)$ annulliert. Enthält dieses Ideal das 1-Element, d.h. stimmt es mit dem Zentrum überein, dann müssen offenbar alle $\operatorname{Ext}_{(T, A)}^{i}(A, M),(i=1,2, \ldots)$ gleich Null sein und folglich ist jeder $\Gamma$-Modul $(\Gamma, \Lambda)$-projektiv [und $(\Gamma, \Lambda)$-injektiv $)^{14}$.

Man erhält hieraus bei Beachtung von (XI) die

Folgerung: Gibt es ein Element $\tau \in Z_{\Gamma}(\Lambda)$ mit $\operatorname{Spur}(\tau)=1_{\Gamma}$, dann gilt

$$
\mathrm{g}-\operatorname{dim}(I) \leqq \mathrm{g}-\operatorname{dim}(\Lambda) .
$$

Ist (5) eine Gruppe, $\mathfrak{S}$ eine Untergruppe vom Index $n$ in (S) und $R$ ein Ring mit 1-Element, dann ist $\Gamma=R[(\mathscr{S}]$ freie Frobenius-Erweiterung von $\Lambda=R[\mathfrak{S}]$. Es folgt, da $n=\operatorname{Spur}(1)$ jeden Modul $\operatorname{Ext}_{(T, A)}^{i}(A, M)$ annulliert und daß im Falle $n R=R$ jeder $\Gamma$-Modul $(\Gamma, \Lambda)$-projektiv ist. Stimmt $\mathfrak{S}$ mit dem neutralen Element von $(S)$ überein, dann sind dies bekannte Resultate. Im Falle, daß $R=Z$ der Ring der ganzen Zahlen ist, erhält man aus unsern Úberlegungen Ergebnisse aus der Kohomologie der Gruppen. Das ist klar, wenn man beachtet, daß für einen Normalteiler $\mathfrak{\mathscr { S }}$ von (SS gilt:

$\operatorname{Ext}_{(Z[\mathfrak{G}], Z[\mathfrak{G}])}^{i}(Z, A)=\operatorname{Ext}_{Z[\mathfrak{G} / \mathfrak{G}]}^{i}\left(Z, A^{\mathfrak{S}}\right)=H^{i}\left(\mathbb{S} / \mathfrak{S}, A^{\mathfrak{S}}\right), \quad(i=0,1, \ldots)$.

Schließlich beweisen wir noch den folgenden bekannten Satz, dessen eine Hälfte sofort aus Satz 4 folgt.

Satz $5^{15}$ : Für einen $\Gamma$-Rechtsmodul $A$ sind die folgenden Eigenschaften äquivalent:

(a) $A$ ist $(\Gamma, \Lambda)$-projektiv.

14 Siehe dazu auch [12].

${ }_{15}$ Dies ist Satz 12 aus [9]; später wurde dieser Satz noch einmal von D. G. Higman in [6] mitgeteilt. Er geht auf [4], Satz 1 zurück, den er als Spezialfall enthält. Im Spezialfall eines Gruppenrings $\Gamma$ siehe z. B. auch [2], S. 233, Prop. 1.1. Der folgende Beweis weicht zum Teil von der Beweisführung in der Literatur ab. 
(b) A ist $(I, A)$-injektiv.

(c) Es gibt ein $g \in \operatorname{Hom}_{A}(A, A)$ mit $\operatorname{Spur}(g)=1_{A}$.

Beweis: Wie schon festgestellt, sind (a) und (b) äquivalent. Ist (c) erfüllt, dann folgen (a) und (b) nach Satz 4. Es bleibt zu zeigen, daß (c) aus (a) folgt. Für $A \otimes \Gamma$ betrachten wir die Abbildung $1_{A} \otimes \psi$, wobei $\psi$ der nach 2.3 existierende Frobenius-Homomorphismus von $\Gamma$ in $\Lambda$ ist. Dann folgt wegen der Dualität der Basen und (9) $\operatorname{Spur}\left(1_{A} \otimes \psi\right)=1_{A} \otimes \Gamma$. Da unter Voraussetzung von (a) $A$ (bis auf Isomorphie) $\Gamma$-direkter Summand von $A \otimes_{A} \Gamma$ ist, hat die Einschränkung $g$ von $1_{A} \otimes \psi$ auf $A$ die gewünschte Eigenschaft.

\section{Literatur}

[1] Artin, E.: Kohomologie endlicher Gruppen. Vorlesungsausarbeitung Math. Sem. Hamburg 1957. - [2] Cartan, H., and S. Eileniberg: Homological Algebra. Princeton Press 1956. - [3] EilenberG, S., and T. NAKayama: On the dimension of moduls and algebras II. Nagoya Math. J. 9, 1-16 (1955). - [4] GASchürz, W.: Über den Fundamentalsatz von MASCHKE zur Darstellungstheorie der endlichen Gruppen. Math. Z. 56, 376-387 (1952). - [5] Gopalakrishnan, N. S., N. Ramabhadran and R. SRIDHARAN: A note on the dimension of moduls and algebras. J. Indian Math. Soc. 21, 185-192 (1957). - [6] Higman, D. G.: Induced and produced moduls. Canadian J. Math. 7, 490-508 (1955). - [7] Hirata, K.: On relative homological algebra of Frobenius extensions. Nagoya Math. J. 15, 17-28 (1959). - [8] HоснsснiLd, G.: Relative homological algebra. Trans. Amer. Math. Soc. 82, 246-269 (1956). - [9] KASCH, F.: Grundlagen einer Theorie der Frobeniuserweiterungen. Math. Ann. 127, 453-474 (1954). - [10] KASCH, F.: Ein Satz über Frobeniuserweiterungen. Arch. Math. (im Druck). - [11] Nakayama, T. and T. Tsuzuku: A remark on Frobenius extensions and endomorphism rings. Nagoya Math. J. 15, 9-16 (1959). - [12] Shimura, G.: On a certain ideal of the center of a Frobeniusean algebra. Sci. Papers Coll. Gen. Educ. Univ. Tokyo 2, 117--124 (1952). 